\title{
Primary cutaneous B-cell lymphoblastic lymphoma in an elderly
} man

\author{
Su-Yeon Cho ${ }^{1}$, Seung-Sook Lee ${ }^{2}$, Dae Hyun Back ${ }^{1}$, Kyung Ah Lim ${ }^{1}$, Ye Rim Lee ${ }^{1}$, Hye Jin Kang ${ }^{1}$ \\ Departments of ${ }^{1}$ Internal Medicine, ${ }^{2}$ Pathology, Korea Cancer Center Hospital, Korea Institute of Radiological and Medical Sciences, \\ Seoul, Korea
}

p-ISSN 1738-7949 / e-ISSN 2092-9129 http://dx.doi.org/10.5045/kjh.2011.46.4.283 Korean J Hematol 2011;46:283-6.

Received on July 4, 2011

Revised on August 31, 2011

Accepted on November 14, 2011

\section{Correspondence to}

Hye Jin Kang, M.D.

Division of Hematology/Oncology,

Department of Internal Medicine, Korea Cancer Center Hospital, 75, Nowon-gil,

Nowon-gu, Seoul 139-706, Korea

Tel: +82-2-970-1289

Fax: +82-2-970-2410

E-mail: hyejin@kcch.re.kr

(C) 2011 Korean Society of Hematology
Precursor B-cell lymphoblastic lymphoma (B-LBL) is an uncommon high-grade neoplasm of immature B cells. It occurs predominantly in childhood with extranodal involvement such as skin and bone. Therefore, primary cutaneous involvement in elderly adults is a very rare manifestation of B-LBL. Here, we report a 78-year-old man with B-LBL presenting as a single cutaneous lesion which was immunohistochemically positive for leukocyte common antigen (LCA), CD79a, paired box 5 (PAX5), B cell lymphoma-2 (bcl-2), and terminal deoxynucleotidyl transferase (TdT) staining, but was without systemic involvement. The patient was treated using cyclophosphamide, adriamycin, vincristine, and prednisolone (CHOP), and achieved complete response (CR) at the first response assessment conducted after $3 \mathrm{CHOP}$ cycles. After an additional cycle of $\mathrm{CHOP}$ treatment, radiotherapy was administered at a total dose of 3,600 cGy over 4 weeks. At the 21-month follow-up, he had maintained CR.

Key Words Precursor B-cell lymphoblastic leukemia-lymphoma, Cutaneous, Adult, CHOP, Radiotherapy

\section{INTRODUCTION}

Lymphoblastic lymphoma (LBL) is a highly aggressive subtype of non-Hodgkin's lymphoma (NHL), and it accounts for about $2 \%$ of all NHLs [1]. The majority of acute lymphoblastic leukemias (ALLs) are of the immature B-cell phenotype, whereas approximately $90 \%$ of LBL are of the immature T-cell phenotype [2, 3].

The natural history of B-LBL has not been well defined, and molecular studies are lacking because the literature is limited to small series and case reports. However, it is known that B-LBL has a predilection for children. In 1 study, a review of previous literatures reporting B-LBL in 105 patients, $64 \%$ (63 of 98) of patients for whom the age at diagnosis was available were younger than 18 years [4]. In Korea, the incidence of B-LBL has been reported to be $11.6 \%$ (42 of 360 cases) in children with NHL [5], and only $0.12 \%$ (1 of 802 cases) in adults with NHL [6]. Patients with B-LBL mostly present with extranodal involvement sparing the bone marrow [4, 7]. The most affected sites are skin (33\%), lymph node (22\%), bone (19\%), and mediastinum (5\%) [4]. Although skin is the most affected site, primary involvement of the skin is a rare manifestation of B-LBL because cutaneous B-LBL usually presents secondary to systemic nodal B-LBL $[8,9]$. Here we report a 78 -year-old man with a single cutaneous lesion of B-LBL at diagnosis.

\section{CASE REPORT}

In September 2009, a 78-year-old man visited the Seoul Veterans Hospital with a dark red-colored mass on the right upper quadrant, which he had had for several months. The mass was approximately $50 \times 60 \mathrm{~mm}$ in size, non-tender, non-itching, and round, and had a smooth surface (Fig. 1A). There were no palpable enlarged lymph nodes or any other skin lesions in any region, including the neck, axilla, and groin. Results of a biopsy specimen suggested lymphoma. He was subsequently transferred to the Korea Cancer Center Hospital for a further work-up.

A complete blood count revealed normal values, as follows; white blood cell count, $6.78 \times 10^{9} / \mathrm{L}$; platelet count, $243 \times 10^{9} / \mathrm{L}$; and hemoglobin, $14.0 \mathrm{~g} / \mathrm{dL}$. Aspartate transaminase was 8 $\mathrm{U} / \mathrm{L}$ (normal range, 4-43 U/L), alanine transaminase, $15 \mathrm{U} / \mathrm{L}$ (normal range, 8-38 U/L); uric acid, $6.7 \mathrm{mg} / \mathrm{dL}$ (normal range, This is an Open Access article distributed under the terms of the Creative Commons Attribution Non-Commercial License (http://creativecommons.org/licenses/by-nc/3.0)
which permits unrestricted non-commercial use, distribution, and reproduction in any medium, provided the original work is properly cited. 

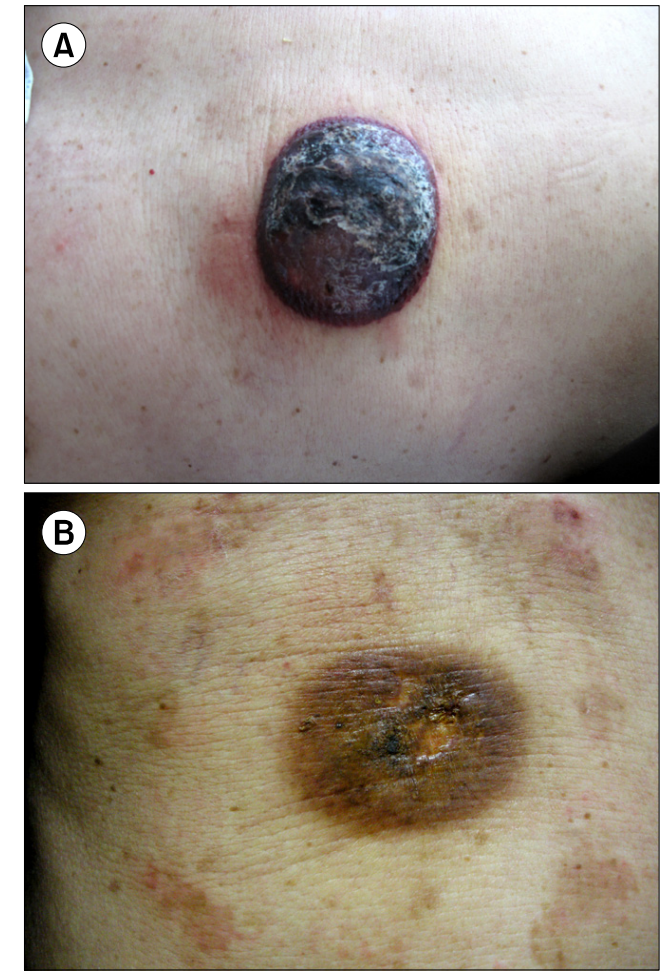
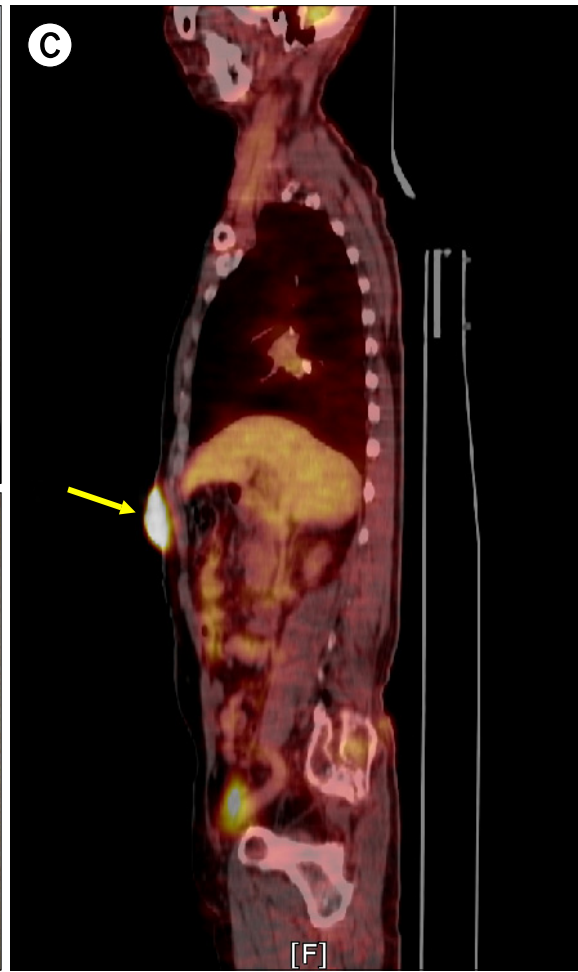

Fig. 1. (A) At diagnosis, a $50 \times 60$ $\mathrm{mm}$ non-tender, non-itching, and dark red tumor with smooth surface was present on the right upper abdominal quadrant. (B) After the $3^{\text {rd }}$ session of cyclophosphamide, adriamycin, vincristine, and prednisolone (CHOP) chemotherapy, the tumor disappeared by gross examination. (C) ${ }^{18} \mathrm{~F}$-fluorodeoxyglucose (FDG) positron emission tomography (PET)/computed tomography (CT) scan shows a single hypermetabolic lesion (arrow) in the abdominal wall (standardized uptake value [SUV]= 12.8).
3.4-7.0 mg/dL); lactate dehydrogenase, $286 \mathrm{U} / \mathrm{L}$ (normal range, 240-480 U/L); and alkaline phosphatase, $57 \mathrm{U} / \mathrm{L}$ (normal range, 40-129 U/L). Computed tomography (CT) of the chest showed normal findings. However, abdominal and pelvic CT scans depicted a 4-cm sized, discoid, highly attenuating mass in the skin of the right upper aspect of the abdomen without demonstrably enlarged lymph nodes. Gastroduodenofiberscopy and colonoscopy produced normal findings. ${ }^{18} \mathrm{~F}$-fluorodeoxyglucose (FDG) positron emission tomography (PET)/CT visualized a single hypermetabolic lesion in the abdominal wall (standardized uptake value $[\mathrm{SUV}]=12.8$ ) (Fig. 1C).

A microscopic examination of the biopsied specimen revealed small malignant cells with fine chromatin, convoluted nuclear membranes, and scant cytoplasm infiltrating the dermis (Fig. 2A, B). On immunohistochemical (IHC) staining, atypical cells showed positivity for B-cell lineage markers (CD79a, paired box 5 [PAX5], and precursor lymphoblastic marker (terminal deoxynucleotidyl transferase [TdT]) (Fig. 2C-F). Staining for CD20 and CD3 was negative. Molecular studies for immunoglobulin heavy chain gene rearrangement also revealed B-cell monoclonality. The final pathological diagnosis was precursor B-LBL.

For staging, bilateral bone marrow (BM) and diagnostic spinal tapping (for cerebrospinal fluid [CSF] cytology) were performed. There was no evidence of lymphoma involvement in $\mathrm{BM}$ or CSF. On the basis of the pathological diagnosis and clinical staging, he was diagnosed as having primary cutaneous precursor B-LBL, Ann Arbor stage IEA (only localized, single extranodal site involvement). He was treated using the cyclophosphamide, adriamycin, vincristine, and prednisolone (CHOP) regimen (cyclophosphamide, 750 $\mathrm{mg} / \mathrm{m}^{2}$; adriamycin, $35 \mathrm{mg} / \mathrm{m}^{2}$; vincristine, $1.4 \mathrm{mg} / \mathrm{m}^{2}$ [maximum $2 \mathrm{mg}$ ] i.v. on day 1; and prednisolone, $100 \mathrm{mg}$ p.o. on days 1-5), which is not an intensive multi-agent chemotherapy, because of his advanced age. For the same reason, the dose of adriamycin in the standard CHOP regimen was reduced from $50 \mathrm{mg} / \mathrm{m}^{2}$ to $35 \mathrm{mg} / \mathrm{m}^{2}$. After the $1 \mathrm{st}$ CHOP cycle, the mass shrank and had a flattened surface, and after the $3^{\text {rd }}$ cycle, the tumor disappeared by gross examination (Fig. 1B). Furthermore, ${ }^{18}$ F-FDG PET/CT failed to depict a hypermetabolic lesion in the abdomen. We concluded that he had achieved complete response (CR) after 3 cycles of CHOP therapy. An additional CHOP cycle was then administered and the initial skin lesion site was subjected to 3,600 cGy of radiotherapy over 4 weeks. At 21 months after completing 4 cycles of CHOP treatment and radiotherapy, the patient was found to have maintained a state of CR.

\section{DISCUSSION}

Primary cutaneous B-LBL involving only the skin without systemic disease appears to occur almost exclusively in children or young adults, and is extremely rare in older patients $[7,10]$. Furthermore, it appears to be predominantly located in the head and neck region $[9,10]$. Thus, the present case report of a single cutaneous lesion of B-LBL in the abdominal wall in a 78-year-old patient expands our knowledge of this disease.

For a differential diagnosis, comprehensive IHC staining 

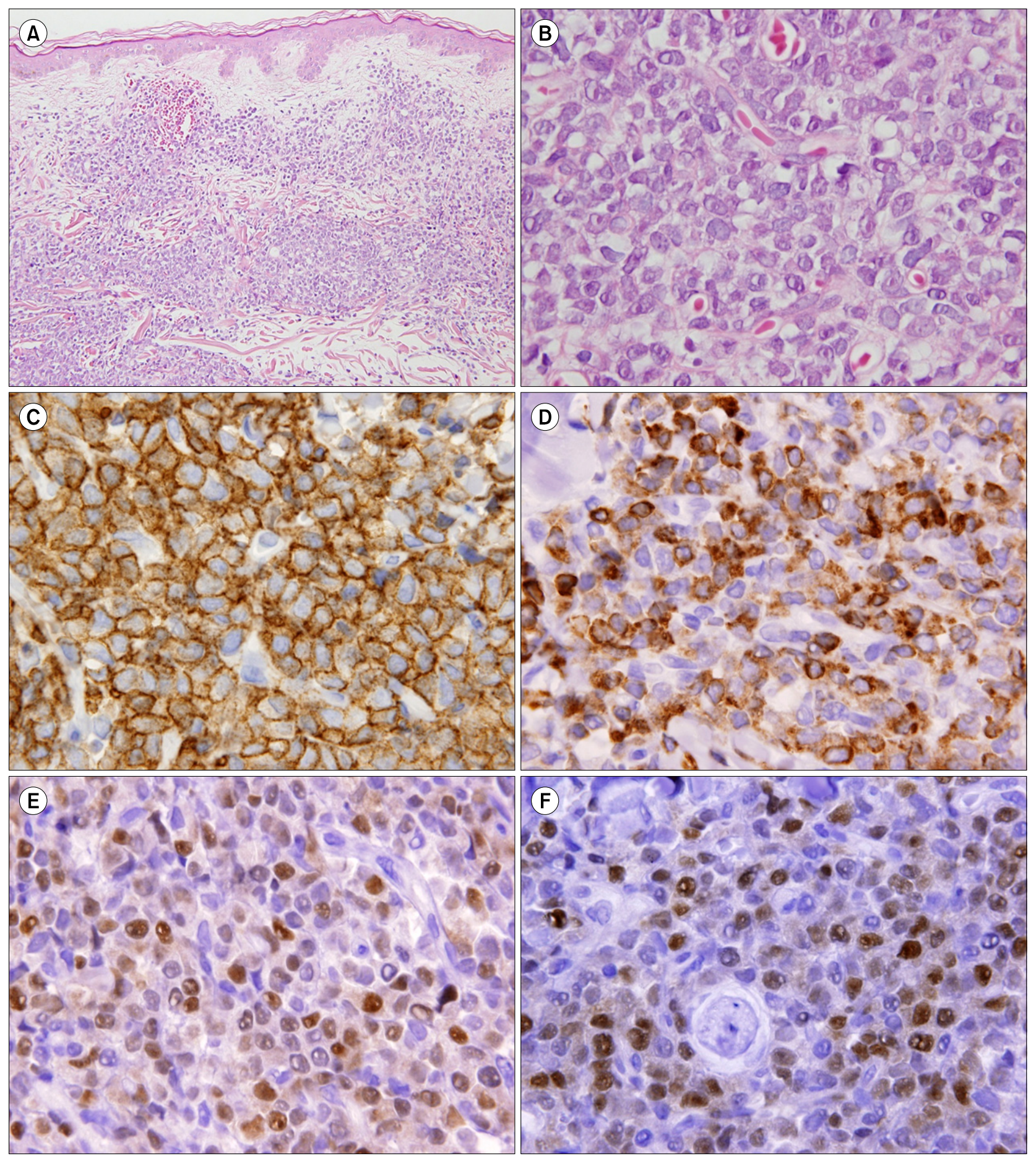

Fig. 2. (A) Microscopically, skin biopsy specimen revealed diffuse infiltration of small to intermediate lymphoid cells in the dermis and subcutaneous fat (hematoxylin \& eosin [H\&E], original magnification $\times 200$ ). (B) High magnification shows monotonous tumor cells with slightly convoluted nuclei with fine chromatin and occasionally nucleolus and scant cytoplasm (H\&E, original magnification $\times 1,000)$. (C-F) Photographs of immunohistochemical staining. Most tumor cells are positive for leukocyte common antigen (LCA) (C), CD79a (D), paired box 5 (PAX5) (E), and terminal deoxynucleotidyl transferase (TdT) (F).

is crucial, but no single diagnostic immunohistologic pattern has been established for B-LBL. Nevertheless, certain immunophenotypic patterns can help distinguish malignant lymphomas and benign lymphoid proliferations. The work-up for the differential diagnosis of B-LBL includes small, blue, round cell tumors, e.g., Ewing's sarcoma, Merkel's cell carcinoma, neuroblastoma, rhabdomyosarcoma, Wilm's tumor, neuroendocrine carcinoma, and metastatic small cell carcinoma, as well as other lymphoid malignant neoplasms [8]. B-lymphoblasts are positive for CD10, 
surface CD22, CD24, PAX5, and TdT in most cases, while the expression of CD20 and CD34 is variable [11]. In tissue sections, CD79a and PAX5 are most frequently used to demonstrate B-cell differentiation, but the former reacts with some cases of T-ALL and, thus, is not specific [12]. In the present case, small malignant cells were positive for LCA, TdT, CD79a, PAX5, and bcl-2, but negative for CD20, CD10, and CD3. These immunohistochemical findings were consistent with precursor B-LBL, although the cells were negative for CD20 and CD10.

Patients with B-LBL respond well to intensive multi-agent chemotherapy (ALL-type regimens or intensive NHL-type regimens) $[4,13]$. According to some reports, adult patients with B-LBL seem to have better CR rates and longer response duration than those with acute B-LBL treated with similar regimens [7]. In addition, it has been suggested that survival is better for B-LBL than T-LBL [13]. Primary cutaneous and extracutaneous B-LBL are not different in the managements; both conditions should be treated with intensive chemotherapy regardless of the staging results $[4,7,13]$. However, our case was treated using the CHOP regimen because of his far advanced age. Morel et al. reported treatment outcomes by different treatment protocols in 80 adult patients with LBL. In that study, the CR rate with CHOP was $71 \%$ compared to $83 \%$ with the intensive NHL protocol (LNH-84) and 86-91\% with 2 different ALL protocols (LALA or FRALLE) [14]. However, there was no significant difference in CR rate and CR duration among the regimens. In addition, there was no significant difference between the CHOP and LNH-84 protocols in overall survival of Ann Arbor stage I to III patients (2-year survival, 62\% and 74\%, respectively; $P=0.49$ ). On the basis of the results demonstrated by Morel et al., we suggest that the CHOP regimen can be a reasonable second option in elderly patient who would not tolerate standard intensive regimens for LBL.

The prognosis of patients with cutaneous LBL appears to be similar to that of patients with extracutaneous LBL [15]. Some authors have reported that a proportion of patients without systemic disease at diagnosis might progress to advanced disease with systemic involvement, most commonly to precursor B-ALL over a period of days to months [9]. These reports suggest that primary cutaneous precursor B-LBL has the potential to progress without adequate systemic chemotherapy, and thus, cutaneous infiltrates might be an early manifestation of precursor B-LBL/lymphoma progression to diffuse disease [9].

In conclusion, we report a case of B-LBL presenting with a single cutaneous lesion without systemic involvement in an old man. This case suggests that the possibility of primary cutaneous B-LBL among several subtypes of cutaneous lymphomas be considered despite its rarity, when an adult patient presents with only skin involvement and has pathological findings compatible with lymphoma.

\section{REFERENCES}

1. The Non-Hodgkin's Lymphoma Classification Project. A clinical evaluation of the International Lymphoma Study Group classification of non-Hodgkin's lymphoma. The Non-Hodgkin's Lymphoma Classification Project. Blood 1997;89:3909-18.

2. Pui CH, Behm FG, Crist WM. Clinical and biologic relevance of immunologic marker studies in childhood acute lymphoblastic leukemia. Blood 1993;82:343-62.

3. Grogan T, Spier C, Wirt DP, et al. Immunologic complexity of lymphoblastic lymphoma. Diagn Immunol 1986;4:81-8.

4. Maitra A, McKenna RW, Weinberg AG, Schneider NR, Kroft SH. Precursor B-cell lymphoblastic lymphoma. A study of nine cases lacking blood and bone marrow involvement and review of the literature. Am J Clin Pathol 2001;115:868-75.

5. Lyu CJ, Kang IJ, Koo HH, et al. Epidemiology and clinical outcomes in children with malignant lymphoma in Korea: retrospective study. Korean J Pediatr Hematol Oncol 2004;11: 153-63.

6. Kang YK, Kim BS, Kim TW, et al. Clinicopathologic characteristics of Korean non-Hodgkin's lymphomas based on REAL classification. J Korean Cancer Assoc 1999;31:641-52.

7. Lin P, Jones D, Dorfman DM, Medeiros LJ. Precursor B-cell lymphoblastic lymphoma: a predominantly extranodal tumor with low propensity for leukemic involvement. Am J Surg Pathol 2000;24:1480-90.

8. Shafer D, Wu H, Al-Saleem T, et al. Cutaneous precursor B-cell lymphoblastic lymphoma in 2 adult patients: clinicopathologic and molecular cytogenetic studies with a review of the literature. Arch Dermatol 2008;144:1155-62.

9. Muljono A, Graf NS, Arbuckle S. Primary cutaneous lymphoblastic lymphoma in children: series of eight cases with review of the literature. Pathology 2009;41:223-8.

10. Kawakami T, Kimura S, Kinoshita A, Kondo K, Soma Y. Precursor B-cell lymphoblastic lymphoma with only cutaneous involvement. Acta Derm Venereol 2009;89:540-1.

11. Swerdlow SH, Campo E, Harris NL, et al, eds. WHO classification of tumours of haematopoietic and lymphoid tissues. 4th ed. Lyon, France: IARC Press, 2008:168-70.

12. Hashimoto M, Yamashita Y, Mori N. Immunohistochemical detection of CD79a expression in precursor $\mathrm{T}$ cell lymphoblastic lymphoma/leukaemias. J Pathol 2002;197:341-7.

13. Soslow RA, Baergen RN, Warnke RA. B-lineage lymphoblastic lymphoma is a clinicopathologic entity distinct from other histologically similar aggressive lymphomas with blastic morphology. Cancer 1999;85:2648-54.

14. Morel P, Lepage E, Brice $\mathrm{P}$, et al. Prognosis and treatment of lymphoblastic lymphoma in adults: a report on 80 patients. J Clin Oncol 1992;10:1078-85.

15. Chimenti S, Fink-Puches R, Peris K, et al. Cutaneous involvement in lymphoblastic lymphoma. J Cutan Pathol 1999;26:379-85. 